\title{
CARBONACEOUS NANOMATERIALS FOR WATER POLLUTION REMEDIATION: AN OVERVIEW
}

\author{
Dr. Shweta Vyas \\ Department of Pure \& Applied Chemistry, \\ University of Kota, Kota, \\ Rajasthan ,India
}

Article DOI: https://doi.org/10.36713/epra6185

\begin{abstract}
Any Material, having structure in nanometer scale with at least one dimension is recognized as Nanomaterial (NM). These Nanomaterial (NMs) have vast variations in their elemental composition; which may be formed by single element viz. Gold, Silver, Iron, Carbon etc. or by the combination with Oxygen or Sulphur as Oxides \& Sulphides viz. Iron oxide (Fe ${ }_{2} \mathrm{O}_{3}$, Copper Oxide (CuO) or Cadmium Sulphide(CdS),etc. Among the single elemental forms, the Carbonaceous Nanomaterials(CNMs) are identified as nanosized allotropic forms of Carbon having different structures like Nano rods, Nano tubes, Nano ring, Nano sphere, and Nano sheets mostly with $\mathrm{sp}^{2}$ carbon atoms. In past decades, Carbonaceous Nanomaterials(CNMs) have gain interest of researchers due to their biocompatibility, non-toxicity and unique surface to volume properties, etc. their easy tunable features are found advantageous for their utilization in wide range of applications. Further, owing to their specific mechanical strength, conductivity, surface activity and many more beneficial features, they have vast range of applicability in electronic, biomedical, energy storage \& conversion, catalysis and remediation of environmental pollution. Present paper briefly describes about the types of Carbonaceous Nanomaterials (CNMs) with emphasizing their use in the remediation of Water Pollution. With the growing development of Nanotechnologies, use of CNMs may play a significant role in remediation of Water Pollution.
\end{abstract}

KEYWORDS: Carbonaceous Nanomaterials(CNMs), Water Pollution, Remediation of Pollution.

\section{INTRODUCTION}

Water, the most valuable resource available on our planet for the survival of all living beings is depleting continuously with growing population and getting contaminated by industrial or anthropogenic activities. The adverse effect of environmental pollution is observed not only on human health but it also affects the overall economy of the country [1]. Around the globe, more than four billion people are facing water scarcity which may be seen as baseline water stress presented in Figure1 [2] -

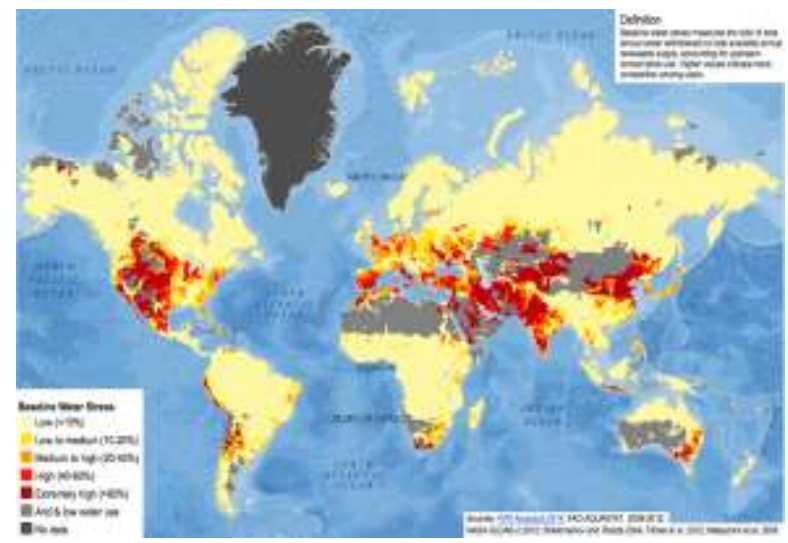

Thus, it is an urgent need to look for some advance technologies which can effectively remove various pollutants from water with ease of bulk availability of the raw material used. Many researchers choose Carbon as raw material, since it is the sixth most abundantly found element on our earth with unique power of self catenation; capable of giving numerous allotropic forms. Among all the allotropic forms, Graphite and Diamond are the historically well known famous forms while many other modern forms viz. Grapheme, Fullerene, Carbon Nanotubes etc. are recognized later on. Carbonaceous Nanomaterials (CNMs) may be defined as any allotropic form of Carbon, having any structural unit with at least one dimension in nanoscale range. The shape and size of these CNMs may be fibrous, tubular, flat sheet, highly porous, or some other types, which may vary significantly with the preparative method adopted. The large surface to volume ratio with unique mechanical, physical and chemical properties make them useful for wide range of applications viz. Environmental Pollution, Super capacitor, Energy storage, etc. In present study, we will discuss about the environmental remediation by $\mathrm{CNMs}$ through various processes as given in Fig 1.

Figure 1 Baseline Water Stress [2] 


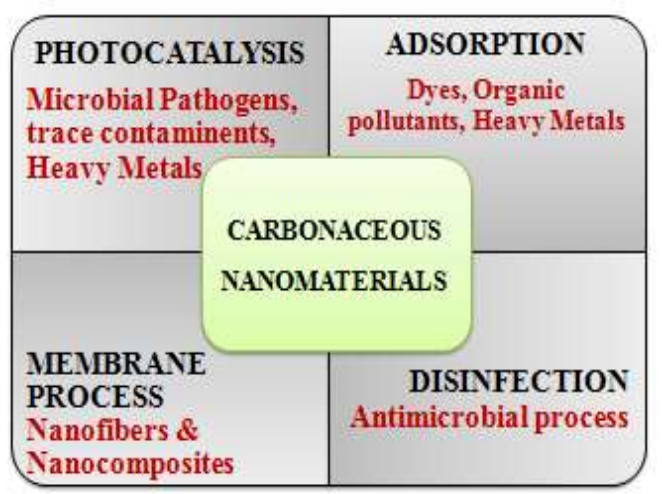

Figure 2 Application of Carbonaceous Nanomaterials in water treatment

\section{CLASSIFICATION OF CNMS \& MECHANISM OF ACTION}

Nanomaterials may be classified on the basis of shapes, size, structure and dimensions. Most broadly accepted classification is on the basis of dimensional characteristics viz. 0D, 1D, 2D, 3D, here the DDimension refer to the number of dimensions where any material is not within the size limit of $0.1-100 \mathrm{~nm}$ [3], on this basis CNMs may be classified as shown in Figure 3-

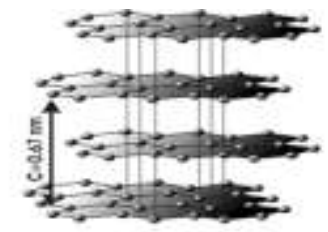

3D

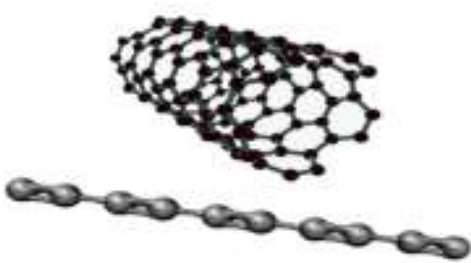

1D

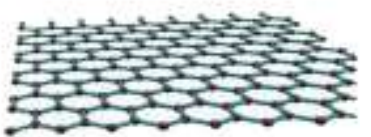

2D

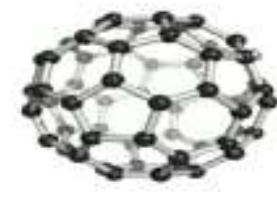

OD
Figure 3 Fullerene (0D),CNT(1D), Graphene(2D) and Graphite (3D) Various Dimensions of CNMs [4]

There are numerous methods available for purification of water like coagulation, extraction, ion exchange, reverse osmosis, filtration, precipitation and adsorption etc. Adsorption [5] is considered as the best method because it is comparatively cost-effective, highly efficient and easy method. Carbonaceous Materials at nanoscale dimensions are found to be very effective Nanosorbents [6] due to their high porosity, large surface area and biocompatibility. However, surface modification is commonly performed by researchers to increase their solubility, reactivity and adsorptive capacity of these materials. Generally chemical treatment by acid, base, oxidizing agents, reducing agents, metals, metal oxides etc. is carried out for this purpose [7]. Mechanism of adsorptive action of CNMs is extremely complex and depends upon the individual interaction of adsorbentadsorbate interactions i.e. targeted pollutant and surface active functional groups available on CNMs, the action may be ionic, covalent, weak inter-atomic attractions or combination of any of these. Thus, individual type of CNM surface and pollutant interactions control the mode of action of targeted molecule to be removed from matrix that is briefly discussed with each type of CNM-

\subsection{Fullerenes}

Fullerenes are recognized as the hollow spherical cage shaped zero dimensional (0D) allotropic form of Carbon having a fixed number of hexagonal \& pentagonal arrangements of $\mathrm{SP}^{2}$ carbon atoms in extremely symmetrical manner known as "Buckyballs" with varying numbers of carbon atoms from as low as 20 upto highest 300 atoms. Fullerens are discovered in 1985 by Harold. W. Kroto, Robert F. Curl and Richard E. Smalley, for which they received Nobel Prize in Chemistry for the year 1996 [8]. The unique structure of Fullerene is described in the form of $\mathrm{C}_{60}$ which is the most common form of fullerene termed as Buckminsterfullerene having 12 pentagons and 20 hexagonal rings of carbon looking like a soccer ball [9].

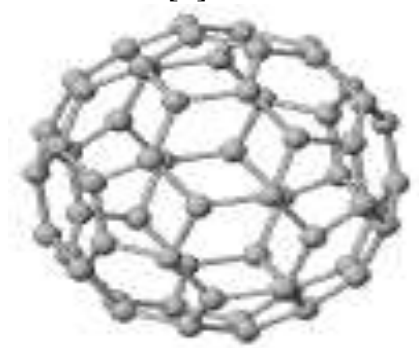

Figure $4 \mathrm{C}_{60}$ Buckminster Fullerene [25].

Fullerene is slightly soluble in water, however nanoscale

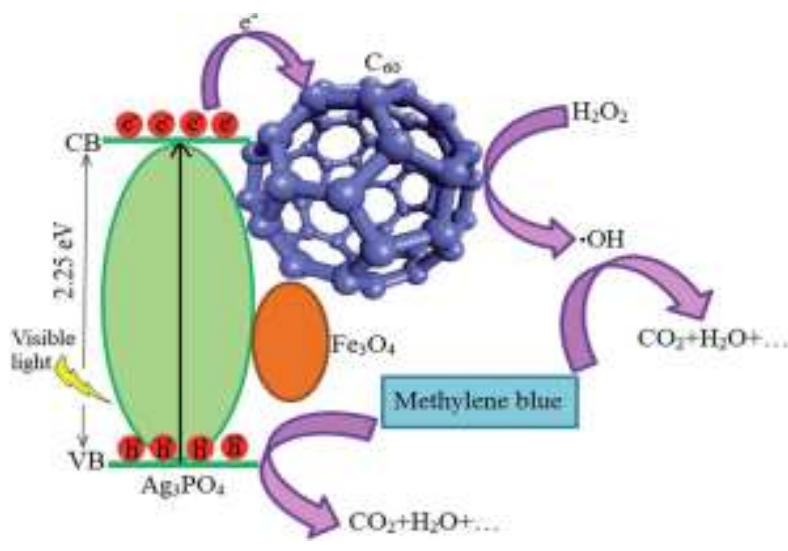

water soluble aggregates of fullerene derivatives were 
reported by encapsulating it with some hydrophilic molecules to develop it as an antibacterial for the disinfection and microbial control [10] It is reported elsewhere that derivatization or modification of Fullerene surface by suitable molecules can create many active sites Figure 5 Mechanism of action of Fullerene nanocomposite material.

on fullerenes for the removal of toxic heavy metal ions from waste water [11]. Fullerene ternary nanocomposites have been used as efficient photo catalytic reagent with antibacterial properties to remove dye and pathogenic pollutants[12]. $\mathrm{C}_{60}$ form of Fullerene has relatively very poor solubility, miscibility, and process ability, thus to apply it in various fields much more concern must be given to undress it. Hence fullerene may open up a new research attempts for application in wastewater treatment.

\subsection{Carbon Nano Tubes (CNT)}

Nanoscale carbon having needle shape tubular structure with $\mathrm{sp}^{2}$ carbon atoms was discovered by Sumio Iijima
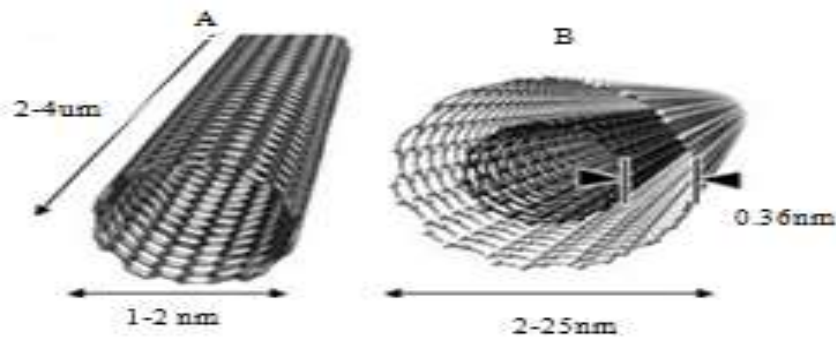

Figure 6 (A). Single walled CNT (B). Multi-walled CNT

and called as Carbon Nanotubes (CNT) [13].CNT are further divided in to two types-Single-Walled CNT(SWCNT) and Multi-Walled CNT(MWCNT) having graphitic carbon atoms in single layer with closed edges or multiple layers respectively, possessing cylindrical tube like structure with diameter of nanometer range and length up to several millimeters as shown in Figure 6 [14]. CNT are found to be extremely hydrophobic in nature [15] and thus suitable to capture many non-polar organic pollutants like Poly Aromatic Hydrocarbon (PAH) [16], Chlorophenols[17] and many organic Dyes[18] from polluted water. Modification of surface sites of CNT can be achieved by chemical reactions like oxidation, halogenations, ozone treatment, heat treatment, etc. which may convert CNT surfaces into polar surface for the remediation of any ionic pollutants like Fluoride, Nitrate, Mercury, Cadmium, etc. many other hazardous toxic metals[19,20]. The mechanism of removal of pollutants is very complex, it may be sorption occurring by chemical or physical forces, CNTs absorptive cites may be found at three places, firstly at interiors hollow space of nanotubes which are open ended, secondly at interstitial spaces in between the tubes, thirdly at groves present at the boundary of nanotube bundles and the adsorption may occur at the outermost surface of the CNTs [21,22].The removal of charged particles may be attributed to electrostatic attraction forces. Utilization of CNT and surface modified CNT has been reported as an very effective Nanosorbents for the removal of ionic pollutants[23,24] and the adsorptive capacities of functionalized CNT have been found superior results than the normal CNT for the removal of $\operatorname{Lead}(\mathrm{Pb})$ [25], Chromium(Cr) [26], Mercury $(\mathrm{Hg})$ [27] like hazardous heavy metal ions. Polymer functionalized CNT shown greater reactivity for removal of heavy metal ions, graphical mechanism[28] of that is shown in figure 7.

As CNTs has provided a wide range of application in waste water treatment, further modification methods can be used in remediation of many other pollutants.

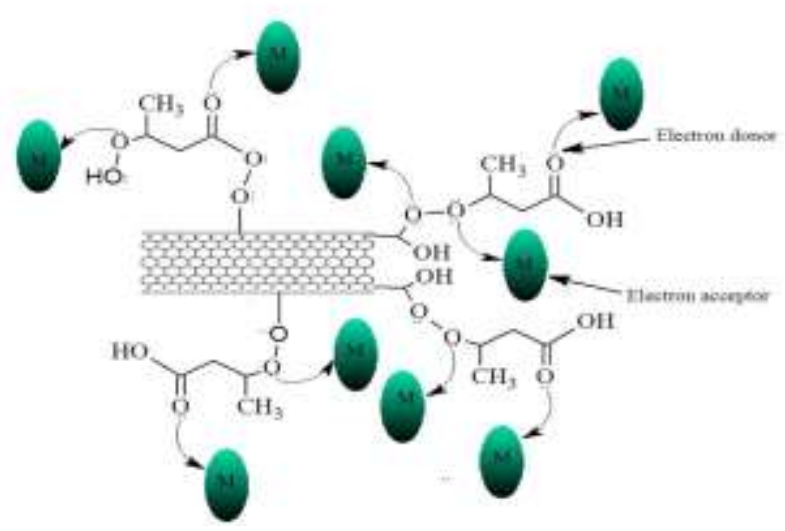

Figure 6 Mechanism of heavy metal removal by functionalized Carbon Nanotube [28]

\subsection{Graphene \& Graphene Oxides}

One atom thick two dimensional sheet of $\mathrm{sp}^{2}$ carbon atoms arranged in hexagonal ring shapes within nano scale range Figure 7 a, b [29], possess amazing mechanical and electrical properties. For the production and characterization of this astounding Nanomaterial Gien and Novoselov [30] received Noble Prize in physics for the year 2010. Owing to its specific electrical and mechanical properties initially Graphene has been used only for electronic applications in super capacitors and transistors [31] but later on it has been reported as an effective solution for desalination of saline water in the form of membrane filtration [32] with cost effective approach.
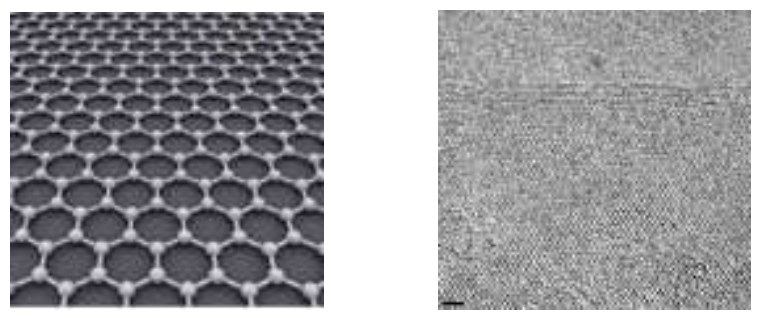

Figure 7 a (i) Computerized Structure of Graphene, [16] b (ii) Atomic resolution imaging of Graphene membranes[16]

A group of researchers obtained nanocomposites of Graphene by the bioreduction of Graphene Oxide and 
intercalated silver ion has been reported as an effective nanosorbent for pathogenic effluent treatment [33].<smiles>CC(C)(C)NC(=O)C1=CC(=CC=O)CC(C(=O)O)N1</smiles>

Betalamic acid
(Amaranthuspolygonoides)

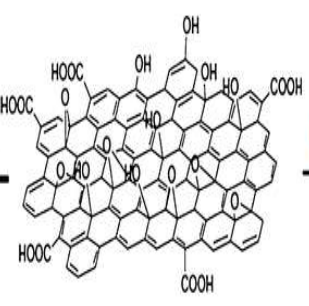

Graphene oxide (GO)

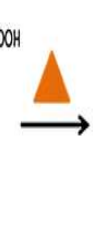

Reduced Graphene oxide (RGO)

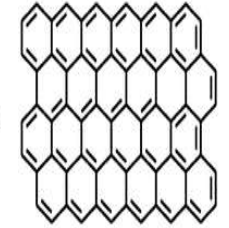

Figure 8 Bioreduction of Graphene oxide by Amaranthus plant extract

Adsorptive removal of many organic pollutants viz. dyes, drugs, toxic chemicals, pesticides and oils has also been reported in literature, with explaining possible mechanisms of removal of organic pollutants by the graphene-based materials involving $\pi-\pi$ interaction, anion$\pi$ interaction and cation- $\pi$ interaction and weak functional group interactions [34]. Tea Poly phenols modified grapheme nanosheets have been reported as an effective sorbent for lead ion removal with antibacterial properties and greater selectivity over other metal ions with ease of regeneration [35]. Oxidized form of Grapheme is well known as Grapheme oxide(GO), having many oxygen bearing functional groups on its surface viz. Carbonyl, carboxylic, Epoxy etc. [36], with the help of these functional groups many Graphene Oxide layers can stack upon each other to form a thin film or filtration membrane[37] which are found to be extremely useful in Nano filtration techniques for wastewater treatment [38]. The large specific surface area of GO make it suitable to adsorb higher amounts of pollutants on it's surface [39]. GO based hydrogel is also reported in literature and proved to be superior adsorbent for the removal of dyes with the ease of separation from aqueous phase[40].

\subsection{Nano Activated Carbon (NAC)}

Amorphous structural form of Nano Carbon with average particle size less than $100 \mathrm{~nm}$ comes under this category of allotropic form of Carbon. Structure of NAC is complex, highly porous, having large surface area with minute amount of other elements like Oxygen, Silica or some other metals or ash [41]. Nano Activated Carbon has highly porous structure with pore size varying from less than nanometer to some hundred nanometers [42] as shown in figure 9-

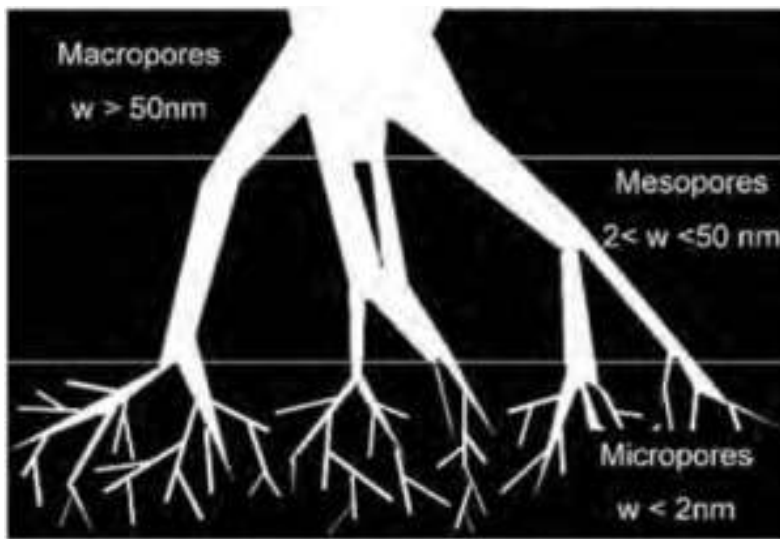

Figure 9 Graphical Distribution of Nano-Pores in NAC [41].

The nanoporous Carbon is found to be super-active for adsorptive removal of phenol [43] and some heavy metal ions [44]. Impregnation of other metallic nanoparticles on NAC was reported to be more effective adsorbent for the simultaneous removal of many heavy metal ions [45]. Recently NAC was prepared in cost effective manner from agro-waste products for the removal of Dye stuffs [46] and ferrous ion [47] from industrial effluents. It is observed that removal of heavy metals by NAC depends on the distribution of porosity, oxygen functional groups, surface area etc. found on NAC [48]. Surface modification improves the adsorption capacity and it is found that NAC modified with magnetic nano metal oxides can regenerated efficiently [49], consequently further research efforts must be imparted for the development of various types of metal oxides embedded NAC. Recently, a group of scientists explained the complicated mechanism of adsorptive removal of Phenols \& Nitro-Phenols using agrowaste derived NAC [50].

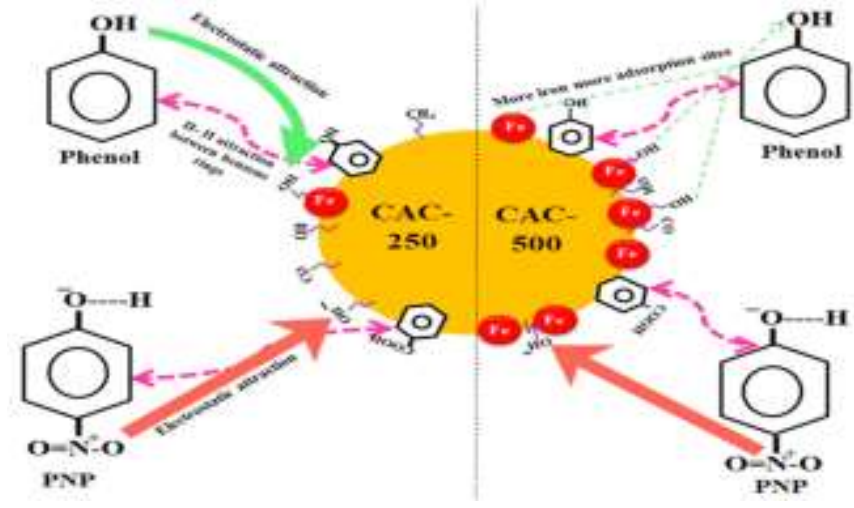

Figure 10 Removal of phenol \& p-Nitro Phenol by NAC [50].

\section{CONCLUSION}

On the basis of the fundamental structure, shape and dimension classification of Carbonaceous Nanomaterials (CNMs) is briefly discussed with giving brief emphasis on mechanism of action and some applications of CNMs in 
water pollution remediation. We hope that it can be helpful for pioneer researchers, working in the field of Nanomaterials. However the impact of Nanometerials on environment and our future generations including overall ecosystem must be taken into considerations for the sake of safety purposes.

\section{REFERENCES}

1. Yaqoob, A.A., Parveen, T., Umar, K., Mohamad Ibrahim, M.N. (2020) "Role of Nanomaterials in the Treatment of Wastewater: A Review". Water, 12, 495.

2. https://en.wikipedia.org/wiki/Water scarcity\#cite_note-1

3. Dong $Y, L i ~ F$ and Wang $Y$, (2020) "Low-Dimension Nanomaterial-Based Sensing Matrices for Antibiotics Detection: A Mini Review." Front. Chem. 8:551, doi: 10.3389/fchem.2020.00551.

4. https://epm.univie.ac.at/research/low-dimensional-quantumsolids/research-focus/pristine-carbon-nanostructures/

5. Czikkely $M$ et al(2018), "Review of heavy metal adsorption processes by several organic matters from wastewaters". Water 10(10):1377.

6. Sweetman M. J., May S., Mebberson N., Pendleton P. Vasilev K. , Plush S. E. and Hayball J. D., (2017) “Activated Carbon, Carbon Nanotubes and Graphene: Materials and Composites for Advanced Water Purification" Journal of Carbon Research, 3, 18; doi:10.3390/c3020018.

7. Coville NJ, Mhlanga SD, Nxumalo EN, Shaikjee A. (2011), “A review of shaped carbon nanomaterials." S. Af.r J Sci. ,107(3/4).

8. https://www.nobelprize.org/prizes/chemistry/1996/summary/

9. Yadav B.C. and Kumar Ritesh.(2008) "Structure, properties and applications of fullerenes". International Journal of Nanotechnology and Applications, 2, 15-24.

10. Lyon D. Y., Brown D. A. and Alvarez P. J. J., .(2008), "Implications and potential applications of bactericidal fullerene water suspensions: effect of nC60 concentration, exposure conditions and shelf life". Water Science and Technology, 57.10, 1533-1538.

11. Sharma, A., Das, J. .(2019) "Small molecules derived carbon dots: synthesis and applications in sensing, catalysis, imaging, and biomedicine". J Nanobiotechnol 17, 92.

12. S. Sepahvand S. and Farhadi S., .(2018)Fullerene-modified magnetic silver phosphate(Ag3PO4/Fe3O4/C60) nanocomposites:hydrothermal synthesis, characterization and study of photocatalytic, catalytic and antibacterial activities RSC Adv., 8, 10124-10140

13. Lijima S.,(1991) "Helical Microtubules of Graphitic Carbon”. Nature, 354, 56-58.

14. Lijima S. \& ToshinariI.(1993) "Single-shell carbon nanotubes of 1-nm diameter". Nature, 363, 603-605.

15. He Hua, HuygLien Ai Pham, Dramou Pierre, Xiao Deli, Zuo Pengli, and Pham-Huy Chuong. .(2013)"Carbon Nanotubes:Applications in Pharmacy and Medicine”. BioMed Research International, Article ID 578290, 1-12.

16. S, Yang CM, Hattori Y, Takahashi K, Kanoh H, Kaneko K. (2007) "Adsorption of polyaromatic hydrocarbons on single walled carbon nanotubes of different functionalities and diameters". J Colloid Interface Sci., 314, 18-24.

17. Liao Q, Sun J, Gao L. .(2008) "Adsorption of chlorophenols by multi walled carbon nanotubes treated with $\mathrm{HNO}_{3}$ and $\mathrm{NH}_{3}{ }$ "Carbon, 46, 544-561.

18. Rajabi M., Mahanpoora K. and Moradi O., (2017) "Removal of dye molecules from aqueous solution by carbon nanotubes and carbon nanotube functional groups: critical review." RSC Adv., 7, 47083-47090.

19. Li YH, Wang S, Zhang X, Wei J, Xu C, Luan Z, et al. (2003) "Adsorption of fluoride from water by aligned carbon nanotubes”. Mater Res Bull. , 38, 469-76.

20. Di ZC, Ding J, Peng XJ, Li YH, Luan ZK, Liang J.(2006) "Chromium adsorption by aligned carbon nanotubes supported ceria particles”. Chemosphere, 62, 861-865.
21. Agnihotri S, Mota JPB, Rostam-Abadi M, Rood MJ. (2005) "Structural characterization of single walled carbon nanotube bundles by experiment and molecular simulation". Langmuir, 21, 896-904.

22. Kang S, Herzberg M, Rodrigues DF, Elimelech M. (2008) "Antibacterial effects of carbon nanotubes: size does matter". Langmuir,24, 6409-6413.

23. Ihsanullah F., Al-Khaldi F.A., Abu-Sharkh B., Abulkibash A.M. and Qureshi M.I. (2016) "Effect of acid modification on adsorption of hexavalent chromium (Cr(VI)) from aqueous solution by activated carbon and carbon nanotubes". Desalination and Water Treatment, 57(16), 7232-7244.

24. Li J.X., Chen S.Y., Sheng G.D., Hu J., Tan X.L. and Wang X.K. (2011) "Effect of surfactants on $\mathrm{Pb}(\mathrm{II})$ adsorption from aqueous solutions using oxidized multiwall carbon nanotubes". Chem. Eng. J., 166, 551-558.

25. Jun X.U.Y., Arrigo R., Xi L.I.U. and Sheng S.D. (2011) "Characterization and use of functionalized carbon nanotubes for the adsorption of heavy metal anions". New Carbon Mater, 26, 57-62.

26. Sheikh A.H.E., Degs Y.S.A., Asad R.M.A. and Sweileh J.A. (2011) "Effect of oxidation and geometrical dimensions of carbon nanotubes on $\mathrm{Hg}(\mathrm{II})$ sorption and preconcentration from real waters". Desalination, 270, 214-220.

27. Velickovic Z., Vukovic G.D., A.D., Marinkovic M.S., Moldovan, Grujic A.A.P., Uskokovic P.S. and Ristic M.D. (2012) "Adsorption of arsenate on iron(III) oxide coated ethylenediamine functionalized multiwall carbon nanotubes”. Chem. Eng. J., 18,174-181.

28. Bankole, M.T., Abdulkareem, A.S., Mohammed, I.A. et al. (2019) "Selected Heavy Metals Removal From Electroplating Wastewater by Purified and Polyhydroxylbutyrate Functionalized Carbon Nanotubes Adsorbents". Sci Rep 9, 4475.

29. Jannik C. Meyer, A. K. Geim, M. I. Katsnelson, K. S. Novoselov, T. J. Booth. (2007) "The structure of suspended graphene sheets". Nature, 446, 60-63.

30. Novoselov K. S., Geim A. K. S., Morozov V., Jiang D., Zhang Y., Dubonos S. V., Grigorieva I.V., Firsov A.A. (2004) "Electric Field Effect in Atomically Thin Carbon films". Science, 306, 666-669.

31. Raymond L. D. (2014) "Chemical control of grapheme Architecture". ACS Nano, 8, 9733-9754.

32. Surwade S.P., Sergei S.N., Ivan V.V., Raymond R.U.,Sheng D. (2015) "Water Desalination Using Nanoporous Single-Layer Graphene with Tunable Pore Size. "Nature nanotechnology, 10, 459-464.

33. Firdosh M.J., Lalitha P.,(2016)"Nanosilver-decorated nanographene and their adsorpotion performance in wastewater treatment." Bioresour. Bioprocess, 3, 4-12.

34. M. R. Gandhi, Vasudevan S., Shibayama A., Yamada M., (2016) "Graphene and Graphene-Based Composites: A Rising Star in Water Purification - A Comprehensive Overview" Chemistry Select, 1, 4358-4385.

35. Zheng S, Hao L, Zhang L, Wang K, Zheng W, Wang X, Zhou $\mathrm{X}$, Li W, Zhang L. (2018) "Tea Polyphenols Functionalized and Reduced Graphene Oxide-ZnO Composites for Selective $\mathrm{Pb}^{2+}$ Removal and Enhanced Antibacterial Activity." $J$ Biomed Nanotechnol. Jul 1;14(7):1263-1276.

36. Yi Wei,Yushan Zhang,Xueli Gao,Zhun Ma,Xiaojuan Wang,Congjie Gao. (2018) "Multilayered graphene oxide membranes for water treatment: A review”. Carbon, Elsevier139, 964-981.

37. R.K. Joshi, P. Carbone, F.C. Wang, V.G. Kravets, Y. Su, I.V. Grigorieva, H.A. Wu, A.K. Geim, R.R. Nair. (2014) "Precise and ultrafast molecular sieving through grapheme oxide membranes". Science, 343, 752-754.

38. Chong Jeng Yi, Wang Bo, Mattevi Cecilia, Li Kang. (2018) "Dynamic microstructure of graphene oxide membranes and the permeation flux". Journal of Membrane Science, 549, 385-392.

39. N.V. Medhekar, A. Ramasubramaniam, R.S. Ruoff, V.B. Shenoy. (2010) "Hydrogen bond networks in graphene oxide 
composite paper: structure and mechanical properties". ACS Nano, 4, 2300-2306.

40. Guo H., Jiao T., Zhang Q., Guo W., Peng Q., Yan X. (2015) "Preparation of Graphene oxide Based hydrogels as Efficient Dye Adsorbents for Wastewater Treatment". Nanoscale Research Letters, 10,272, 1-10.

41. Saleh N.B., Nabidul A.R.M., Joseph H.B., Nirupam A., Jaime P.T., Tara S.A. (2014) "Emergent Properties and Toxicological Considerations for Nanohybrid materials in Aquatic System”. Nanomaterials, 4(2) 372-407.

42. Shivashankar M. and Bubanale Sadashiv, (2017) "History, Method of Production, Structure and Applications of Activated Carbon". International Journal of Engineering Research \& Technology, 6(6), 495-498.

43. Hwang H., Sahinb O. and Choi Joon W., (2017) "Manufacturing a super-active carbon using fast pyrolysis char from biomass and correlation study on structural features and phenol adsorption". RSC Adv., 7, 42192-42202.

44. Guo J., Song Y. , Ji Xia. , Ji L. , Cai Lu, Wang Y. , Zhang H. and Song W., (2019)"Preparation and Characterization of Nanoporous Activated Carbon Derived from Prawn Shell and Its Application for Removal of Heavy Metal Ions". Materials ,12, 2411-17.

45. Dinesha B.L., Sharanagouda H., Udaykumar N., Ramachandr C.T. and Dandekar Anil B., (2017) "Removal of Pollutants from Water/Waste Water Using Nano-Adsorbents: A Potential Pollution Mitigation”. Int.J.Curr.Microbiol.App.Sci., 6(10), 4868-4872.

46. Pradhanang R. R., Adhikari L. , Goswami S., Adhikari R., Pradhananga M. Rajbhandari R. , Ariga K. and Shrestha Lok Kumar, (2017) "Dye Adsorption on Nanoporous Carbon Materials Derived from Agro-Product.Journal of Carbon Research", 3 (12), 1-11.

47. Thilagam G., Mani N., Arivoi S., Hema M. (2016) "Adsorption behavior of $\mathrm{Fe}$ (II) ion from aqueous solution onto nano carbon". International Journal of Chemical Studies, 4(1),96-102.

48. Gallios George P., Tolkou Athanasia K., Katsoyiannis Ioannis A., Stefusova K., Vaclavikova M. and Deliyanni Eleni A. (2017) "Adsorption of Arsenate by Nano Scaled Activated Carbon Modified by Iron and Manganese Oxides". Sustainability, 9-1684,1-18.

49. Jain M., Yadav M., Kohoutc T., Lahtinen M., Garg V.K., Sillanpääa M., (2018) “ Development of iron oxide/activated carbon nanoparticle composite for the removal of $\mathrm{Cr}(\mathrm{VI})$, $\mathrm{Cu}(\mathrm{II})$ and $\mathrm{Cd}(\mathrm{II})$ ions from aqueous solution" Water Resources and Industry, 20,54-74.

50. Yadav N., Maddheshiaya D. N., Rawat S., Singh J. (2020) "Adsorption and equilibrium studies of phenol and paranitrophenol by magnetic activated carbon synthesised from cauliflower waste." Environ. Eng. Res. 25(5): 742-752. 